\title{
ANÁLISE DISCRIMINANTE DE SOLOS SOB DIFERENTES USOS EM ÁREA DE MATA ATLÂNTICA A PARTIR DE ATRIBUTOS DA MATÉRIA ORGÂNICA ${ }^{1}$
}

\author{
Vinicius de Melo Benites², Rondinele de Oliveira Moutta ${ }^{3}$, Heitor Luiz da Costa Coutinho ${ }^{2}$ e Fabiano \\ de Carvalho Balieiro ${ }^{2}$
}

\begin{abstract}
RESUMO - Na região serrana do Estado do Rio de Janeiro (bioma Mata Atlântica), pequenos agricultores praticam agricultura itinerante no sistema de corte e queima. Neste trabalho, amostras de horizontes superficiais ( 0 -15 cm) de um Latossolo Vermelho-Amarelo sob cinco diferentes coberturas vegetais (Mata Atlântica, cultivo anual, café, banana e pastagem) foram coletadas para caracterização química dos teores de carbono nas diferentes frações de substâncias húmicas. As amostras obtidas sob mata e sob pastagem puderam ser nitidamente isoladas das demais pelo modelo discriminante construído. Aquelas representativas do grupo das culturas (banana, café e cultivo) foram superposicionadas, indicando haver semelhança entre os atributos relativos à matéria orgânica nos solos sob esses usos. O modelo obtido permitiu classificar corretamente $88 \%$ das amostras analisadas. Os atributos ácidos fúlvicos (AF), carbono orgânico (C), nitrogênio total (N) e relação C/N foram selecionados pelo modelo, sendo o teor de ácidos fúlvicos o atributo de maior peso relativo. Esse resultado indica que o fracionamento de substâncias húmicas permite a observação de alterações no solo que não são possíveis de serem identificadas pela simples determinação do teor de carbono orgânico total. Pelo padrão de agrupamento das áreas (mata-pastagem e banana-café-cultivo), denotou-se que o uso de fertilizantes pode se relacionar com alterações em atributos indicadores importantes, como o teor de ácidos fúlvicos.
\end{abstract}

Palavras-chave: Mata Atlântica, Matéria orgânica do solo, Qualidade do solo e Análise multivariada.

\section{DISCRIMINANT ANALYSIS OF SOILS UNDER DIFFERENT LAND USES IN THE ATLANTIC RAIN FOREST AREA USING ORGANIC MATTER ATTRIBUTES}

\begin{abstract}
In the mountain region of the State of Rio de Janeiro (Atlantic Rain Forest biome), small farmers practice shifting cultivation in the slash and burn system. In this work, soil surfaces samples $(0-15 \mathrm{~cm})$ of a Yellow Red Latossolo under five different vegetal coverings (Atlantic Rain Forest, annual culture, coffee, banana and pasture), had been taken in the small farming area of Bom Jardim RJ for soil chemical characterization and carbon content analysis in the different humic fractions. The samples taken under forest and pasture could be clearly isolated from the other groups for the discriminante model created. For the banana, coffee and culture groups, a certain overlapping was observed, indicating a similarity of the soil organic matter attributes between these coverings. The model allowed to correctly classify $88 \%$ of the analyzed samples. The fulvic acid (AF), organic carbon (C), total nitrogen $(N)$, and the $C / N$ ratio had been selected by the model, with the fulvic acids having the attribute of higher relative weight. This result suggests that the humic acids fractionation allows the detection of alterations in the soil that are not possible to be identified for the simple determination of the total organic carbon. The discriminate pattern of areas (forest-pasture and banana-coffe-culture) denotes that fertilizer could be related with changes in some important indicator attributes such as fulvic acids.
\end{abstract}

Keywords: Atlantic Rain Forest, Soil organic matter, Soil quality and multivariate analysis.

\footnotetext{
${ }^{1}$ Recebido em 08.10.2007 e aceito para publicação em 02.03.2010.

22Embrapa Solos, Rio de Janeiro RJ, Brasil.E-mail: <vinicius@cnps.embrapa.br>, <heitor@cnps.embrapa.br>e < balieiro@cnps.embrapa.br>.

${ }^{3}$ Escola de Engenharia de Lorena/USP, Lorena SP, Brasil. E-mail: <romoutta@yahoo.com.br>.
} 


\section{INTRODUÇÃO}

A matéria orgânica do solo(MOS)é componente-chave em qualquer ecossistema terrestre. Variações em sua abundância e, ou, composição podem causar efeitos sobre processos ecossistêmicos e serviços ambientais, como: a capacidade de troca catiônica, a estruturação e retenção de umidade dos solos e a própria capacidade do solo em estocar $\mathrm{C}$ atmosférico fixado pelas plantas. As substâncias húmicas, por sua vez, constituem o principal componente da matéria orgânica dos solos (STEVENSON, 1996).

As características da MOS são resultantes dos processos aos quais os solos foram e estão submetidos, e por isso vários de seus componentes podem ser utilizados como indicadores de mudança de uso do solo (GREGORICH et al., 1994; HAYNES et al., 2000; DENEF et al., 2008). Partindo-se da hipótese de que as frações das substâncias húmicas também refletem as mudanças ocorridas por alterações antrópicas e ao mesmo tempo são estáveis diante das variações espaciais e temporais de curto prazo, em comparação com alguns indicadores biológicos e bioquímicos normalmente avaliados, a caracterização dessas frações apresenta grande potencial na avaliação de alterações na qualidade do solo.

A análise das informações geradas a partir da caracterização química e física de solos pode ser realizada utilizando-se técnicas estatísticas de análise multivariada. A análise multivariada é importante ferramenta para a análise exploratória de dados de solos, permitindo o agrupamento de amostras segundo sua similaridade e ainda permitindo a seleção de variáveis de maior importância na discriminação de grupos pré-selecionados. A utilização dessa ferramenta tem sido observada na literatura internacional em estudos de pedologia e na interpretação de bancos de dados sobre solos (NORRIS, 1971; BURROGHe WEBSTER, 1976). Mais recentemente, Tótola e Chaer (2002) e Balieiro et al. (2005) demonstraram o potencial dessas ferramentas na ordenação de áreas de florestas plantadas sob diferentes manejos e em regeneração natural (áreas de pousio), em função de uma série de atributos físicos, químicos, biológicos e bioquímicos do solo, para avaliação da qualidade do solo e dos substratos degradados.

O Estado do Rio de Janeiro está localizado no bioma Mata Atlântica. Na região serrana do Estado, áreas de Mata Atlântica são utilizadas por pequenos agricultores que praticam agricultura itinerante nos sistemas de corte e queima (EMBRAPA, 2004). O uso agrícola desse sistema que incorpora o pousio ou o descanso da terra ao manejo da área tem sido responsável por manter produtivas áreas acidentadas por longos períodos (RESENDE et al., 2002; REIS, 2002). Contudo, a abertura de áreas com vegetação nativa e o uso agrícola dessas terras com diferentes coberturas vegetais promovem alterações nos atributos do solo e, principalmente, nas características da matéria orgânica do solo. Esse trabalho testou a hipótese de que, a partir da análise das características da MOS, extraídas de amostras de solos sob diferentes usos, é possível separar ou agrupar áreas sob diferentes usos e, assim, auxiliar na mensuração e predição de práticas que podem ser consideradas benéficas ou prejudiciais à manutenção de funções importantes do agroecossistema. A seleção de quais características da MOS são responsáveis por esse agrupamento poderá nortear a escolha de indicadores sensíveis à mudança de uso da terra na região.

\section{MATERIAL E MÉTODOS}

Foram coletadas cinco amostras de horizontes superficiais (0-15 cm) de um Latossolo Vermelho-Amarelo sob diferentes coberturas vegetais em uma área de agricultura itinerante no Município de Bom Jardim RJ, na Mata Atlântica em estágio avançado de sucessão (mata com aproximadamente 150 anos); em área recémaberta para cultivo anual pelo sistema de corte e queima (cultivo); em área sob café com 12 anos (café); área sob banana com 8 anos (banana); e em área sob pastagem com capim-bermuda com 25 anos (pastagem). As amostras de solo após secas ao ar foram destorroadas e peneiradas, obtendo-se a fração com diâmetro menor que $2 \mathrm{~mm}$ (TFSA). Na TFSA foram determinados teores de $\mathrm{Ca}^{2+}, \mathrm{Mg}^{2+}, \mathrm{K}^{+}$e $\mathrm{Na}^{+}$trocáveis; $\mathrm{Al}^{3+}$ extraível, $\mathrm{H}^{+}$, e $\mathrm{pH}$ em água, foram calculadas a soma de bases (S) e a capacidade de troca catiônica (T). Foram ainda determinados os teores das frações areia, silte e argila. As análises de rotina foram realizadas no Laboratório de Análise de Solo e Planta - Embrapa Solos, utilizando-se os métodos descritos em Embrapa (1997).

Entre os atributos relativos à matéria orgânica do solo foram determinados os teores de carbono orgânico, nitrogênio total e relação C/N (EMBRAPA, 1997) e o fracionamento de substâncias húmicas (BENITES et al., 2003), obtendo-se os atributos teor de ácidos húmicos (AH) e ácidos fúlvicos (AF), relação $\mathrm{AH} / \mathrm{AF}$ e a soma das duas frações constituindo o extrato húmico total (EHT). 
A partir dos atributos relativos à matéria orgânica do solo (AH, AF, EHT, AH/AF, C, N e C/N), foi montada uma matriz de dados para análise estatística multivariada. A matriz foi submetida à análise estatística, aplicando-se a análise discriminante (MANLY, 1994). Foi utilizando o método de inclusão progressiva passo a passo (forward stepwise), gerando um modelo discriminante formado por duas variáveis ortogonais de máximo poder discriminante (VD1 e VD2). A partir desse modelo foram geradas a matriz de classificação e as funções discriminantes. Foram testadas as correlações entre atributos relativos à matéria orgânica e características físicas e químicas dos solos determinadas na análise de rotina e as correlações entre as variáveis discriminantes e os atributos do solo. Para as análises estatísticas, utilizou-se o programa computacional Statistica 7.0 (STATSOFT, 2005).

\section{RESULTADOS E DISCUSSÃO}

\subsection{Análise discriminante dos sítios sob diferentes usos}

Os valores das características químicas e a análise granulométrica determinados na análise de rotina dos solos (Tabela 1) e os atributos relativos ao fracionamento químico da matéria orgânica do solo (Tabela 2) compuseram a matriz de análise. Os atributos AF, $\mathrm{C}$, $\mathrm{N}$ e relação $\mathrm{C} / \mathrm{N}$ foram selecionados para as variáveis discriminantes geradas (equações 1 e 2), e o modelo gerado pela matriz foi capaz de classificar corretamente $88 \%$ das amostras analisadas (Tabela 3 ), sendo as áreas de Mata, de cultivo e de pastagem classificadas com $100 \%$ de precisão pelo modelo (Tabela 3). O teor de ácidos fúlvicos (fração de maior atividade, menor peso molecular e estabilidade entre as frações húmicas) foi o atributo de maior peso relativo nos modelos, sugerindo que o fracionamento de substâncias húmicas permite a observação de alterações no solo que não são possíveis de serem identificadas pela simples determinação do teor de carbono orgânico total. Esses resultados corroboram os achados de Haynes et al. (2000), que verificaram, entre os vários componentes lábeis da matéria orgânica do solo (carbono da fração leve da matéria orgânica, carbono da biomassa microbiana, C solúvel, N e P orgânicos solúveis em água), que o C solúvel em água apresentou melhores correlações com as mudanças em curso do solo que o próprio C orgânico.

Tabela 1 - Valores médios e desvios (entre parênteses) de atributos físicos e químicos dos solos sob diferentes usos. Table 1 - Average values (standard deviation) of physical and chemical attributes of soils over contrasting uses.

\begin{tabular}{|c|c|c|c|c|c|c|c|c|c|c|c|c|c|c|}
\hline \multirow[t]{2}{*}{ Uso atual } & \multirow{2}{*}{$\begin{array}{r}\mathrm{pH} \\
\text { agua }\end{array}$} & \multicolumn{2}{|c|}{ Areia } & \multirow[t]{2}{*}{ Silte } & \multirow[t]{2}{*}{ Argila } & \multirow[t]{2}{*}{$\mathrm{Ca}^{2+}$} & \multirow[t]{2}{*}{$\mathrm{Mg}^{2+}$} & \multirow[t]{2}{*}{$\overline{\mathrm{K}^{+}}$} & \multirow[t]{2}{*}{$\mathrm{Na}^{+}$} & \multirow[t]{2}{*}{$\mathrm{S}^{1}$} & \multirow[t]{2}{*}{$\mathrm{Al}^{3+}$} & \multirow[t]{2}{*}{$\mathrm{H}^{+}$} & \multirow[t]{2}{*}{$\mathrm{T}^{2}$} & \multirow[t]{2}{*}{$P$} \\
\hline & & grossa & fina & & & & & & & & & & & \\
\hline & & & $-\mathrm{g}$ & $\overline{g^{-1}}$ & 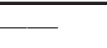 & & & & $-\mathrm{cmol}$ & $\mathrm{kg}^{-1}$ & & & - & $\overline{\mathrm{mg} \mathrm{kg}^{-1}}$ \\
\hline Mata & $\begin{array}{c}4,8 \\
(0,3)\end{array}$ & $\begin{array}{l}506 \\
(74)\end{array}$ & $\begin{array}{c}75 \\
(21)\end{array}$ & $\begin{array}{c}182 \\
(32)\end{array}$ & $\begin{array}{l}236 \\
(28)\end{array}$ & $\begin{array}{c}2,9 \\
(1,6)\end{array}$ & $\begin{array}{c}1,8 \\
(0,9)\end{array}$ & $\begin{array}{c}0,4 \\
(0,1)\end{array}$ & $\begin{array}{c}0,03 \\
(0,01)\end{array}$ & $\begin{array}{c}5,1 \\
(2,5)\end{array}$ & $\begin{array}{c}0,5 \\
(0,5)\end{array}$ & $\begin{array}{c}8,6 \\
(1,4)\end{array}$ & $\begin{array}{l}14,3 \\
(3,2)\end{array}$ & $\begin{array}{c}4,2 \\
(0,5)\end{array}$ \\
\hline Cultivo & $\begin{array}{l}6,5 \\
(0,5)\end{array}$ & $\begin{array}{c}434 \\
(25)\end{array}$ & $\begin{array}{l}117 \\
(12)\end{array}$ & $\begin{array}{l}209 \\
(16)\end{array}$ & $\begin{array}{l}240 \\
(27)\end{array}$ & $\begin{array}{c}7,2 \\
(0,9)\end{array}$ & $\begin{array}{c}1,7 \\
(0,2)\end{array}$ & $\begin{array}{c}1,7 \\
(1,2)\end{array}$ & $\begin{array}{c}0,02 \\
(0,00)\end{array}$ & $\begin{array}{l}10,6 \\
(1,7)\end{array}$ & $\begin{array}{c}0,0 \\
(0,0)\end{array}$ & $\begin{array}{c}2,7 \\
(1,8)\end{array}$ & $\begin{array}{l}13,3 \\
(1,1)\end{array}$ & $\begin{array}{c}119,0 \\
(58,4)\end{array}$ \\
\hline Café & $\begin{array}{c}6,1 \\
(0,2)\end{array}$ & $\begin{array}{l}459 \\
(25)\end{array}$ & $\begin{array}{l}138 \\
(16)\end{array}$ & $\begin{array}{l}176 \\
(6)\end{array}$ & $\begin{array}{l}227 \\
(17)\end{array}$ & $\begin{array}{c}4,8 \\
(1,3)\end{array}$ & $\begin{array}{c}1,3 \\
(0,2)\end{array}$ & $\begin{array}{c}0,8 \\
(0,2)\end{array}$ & $\begin{array}{c}0,02 \\
(0,01)\end{array}$ & $\begin{array}{c}6,9 \\
(1,4)\end{array}$ & $\begin{array}{c}0,0 \\
(0,0)\end{array}$ & $\begin{array}{c}3,9 \\
(0,5)\end{array}$ & $\begin{array}{l}10,8 \\
(1,1)\end{array}$ & $\begin{array}{c}48,4 \\
(14,6)\end{array}$ \\
\hline Banana & $\begin{array}{c}6,0 \\
(0,6)\end{array}$ & $\begin{array}{l}466 \\
(14)\end{array}$ & $\begin{array}{l}106 \\
(9)\end{array}$ & $\begin{array}{l}188 \\
(13)\end{array}$ & $\begin{array}{l}244 \\
(1)\end{array}$ & $\begin{array}{c}6,3 \\
(1,4)\end{array}$ & $\begin{array}{c}1,5 \\
(0,5)\end{array}$ & $\begin{array}{c}0,4 \\
(0,1)\end{array}$ & $\begin{array}{c}0,02 \\
(0,01)\end{array}$ & $\begin{array}{c}8,2 \\
(1,9)\end{array}$ & $\begin{array}{c}0,1 \\
(0,2)\end{array}$ & $\begin{array}{c}3,8 \\
(1,4)\end{array}$ & $\begin{array}{l}12,1 \\
(1,0)\end{array}$ & $\begin{array}{c}37,6 \\
(28,1)\end{array}$ \\
\hline Pastagem & $\begin{array}{c}5,7 \\
(0,1)\end{array}$ & $\begin{array}{l}383 \\
(43)\end{array}$ & $\begin{array}{l}129 \\
(20)\end{array}$ & $\begin{array}{l}211 \\
(34)\end{array}$ & $\begin{array}{l}277 \\
(34)\end{array}$ & $\begin{array}{c}4,0 \\
(0,30\end{array}$ & $\begin{array}{c}1,5 \\
(0,1\end{array}$ & $\begin{array}{l}1,1 \\
(0,3)\end{array}$ & $\begin{array}{l}0,05 \\
(0,0)\end{array}$ & $\begin{array}{c}6,6 \\
(0,5)\end{array}$ & $\begin{array}{l}0,0 \\
(0,1)\end{array}$ & $\begin{array}{c}4,5 \\
(2,5)\end{array}$ & $\begin{array}{l}11,2 \\
(2,6)\end{array}$ & $\begin{array}{c}3,0 \\
(0,0)\end{array}$ \\
\hline
\end{tabular}

${ }^{1}$ Soma de bases e ${ }^{2}$ Capacidade de troca catiônica a pH 7,0.

Tabela 2 - Valores médios e desvios (entre parênteses) de atributos relativos à matéria orgânica dos solos sob diferentes usos.

Table 2 - Average values (standard deviation) of soil organic matter attributes of soil over different uses.

\begin{tabular}{|c|c|c|c|c|c|c|c|c|c|}
\hline \multirow[t]{2}{*}{$\overline{\text { Uso atual }}$} & \multirow[t]{2}{*}{$\mathrm{AH}^{1}$} & $\mathrm{AF}^{2}$ & $\mathrm{EHT}^{3}$ & \multirow[t]{2}{*}{$\mathrm{AH} / \mathrm{AF}^{4}$} & $\mathrm{AH}$ & $\mathrm{AF}$ & $\mathrm{C}$ & $\mathrm{N}$ & \multirow{2}{*}{$\begin{array}{r}\mathrm{C} / \mathrm{N} \\
-\quad \\
\end{array}$} \\
\hline & & $-g$ C & -- & & \multicolumn{2}{|c|}{$-\%$} & \multicolumn{2}{|c|}{$-\mathrm{g} \cdot \mathrm{kg}^{-1}-$} & \\
\hline & $6,(50)$ & $16,1(2$ & 36,7 & 1,27 ( & $56(4)$ & $44(4)$ & 9) & $3,1(1,0)$ & 14,5 \\
\hline & & $(0$ & 8 & 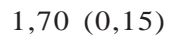 & 63 & ) & 2 & & 2,5 \\
\hline & 8 & $8,8(($ & 3 & 7 & 62 & 3 & & 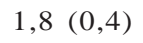 & 12,3 \\
\hline & & 7 , & & 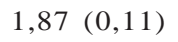 & 6 & & & & 12,0 \\
\hline pastagem & $18,2(2,9)$ & $12,7(0,6)$ & $30,9(3,0)$ & $1,44(0,26)$ & $59(4)$ & $41(4)$ & $35,7(5,1)$ & $2,8(0,3)$ & $12,8(0,8$ \\
\hline
\end{tabular}

${ }^{1}$ Ácidos húmicos, ${ }^{2}$ Ácidos fúlvicos, ${ }^{3}$ Extrato húmico total, ${ }^{4}$ Relação ácido húmico/ácido fúlvico.

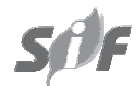

Revista Árvore, Viçosa-MG, v.34, n.4, p.685-690, 2010 
Tabela 3 - Matriz de classificação dos solos sob diferentes usos, segundo o modelo discriminante gerado a partir de atributos relativos à matéria orgânica do solo.

Table 3 - Classification matrix of soils over contrasting uses according to the model obtained using soil organic matter attributes.

\begin{tabular}{lccccc}
\hline Usos & $\begin{array}{c}\text { Corretamente } \\
\text { classificados (\%) }\end{array}$ & mata & cultivo & café & banana \\
\hline mata & 100 & 5 & 0 & 0 & 0 \\
cultivo & 100 & 0 & 5 & 0 & 0 \\
café & 80 & 0 & 0 & 4 & 0 \\
banana & 60 & 0 & 0 & 0 & 3 \\
pastagem & 100 & 0 & 6 & 5 & 0 \\
Total & 88 & 5 & 0 & 5 \\
\hline
\end{tabular}

As duas primeiras variáveis discriminantes acumularam 99,7\% de toda a variância observada pelo modelo, e somente a primeira variável discriminante conteve 93,8\% da variância total (Figura 1). A primeira variável discriminante apresentou correlação negativa $\left(-0,55^{* * *}\right)$ com o teor de ácidos fúlvicos. A segunda variável discriminante apresentou correlação positiva $\left(0,71^{* * *}\right)$ com teor de carbono. As equações das duas variáveis discriminantes foram:

Variável discriminante $1=-1,68 \mathrm{AF}+1,50 \mathrm{C}-12,05$ $\mathrm{N}-3,69 \mathrm{C} / \mathrm{N}+47,33$ (eq. 1 )

Variável discriminante $2=-0,56 \mathrm{AF}-1,64 \mathrm{C}+18,14$ $\mathrm{N}+4,55 \mathrm{C} / \mathrm{N}-42,50$ (eq. 2)

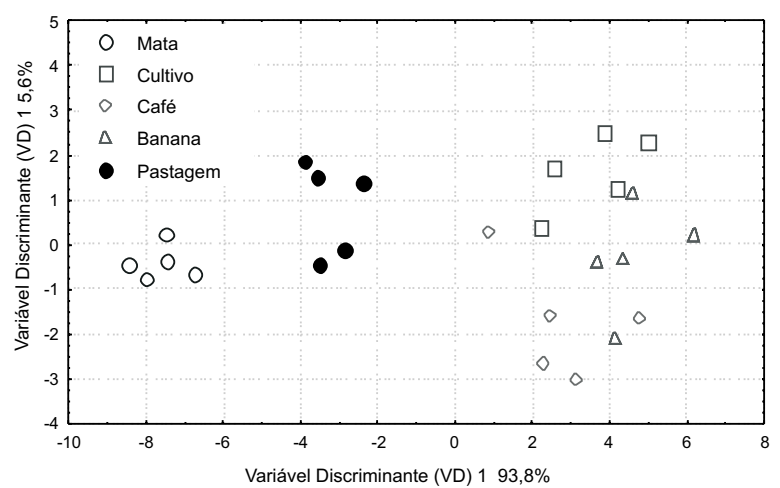

Figura 1 - Gráfico das variáveis discriminantes (VD) segundo o modelo gerado a partir de atributos relativos à matéria orgânica dos solos sob diferentes usos.

Figure 1 - Graph of discriminant variables according to the model obtained using soil organic matter attributes of soil over different uses.

Revista Árvore, Viçosa-MG, v.34, n.4, p.685-690, 2010
As amostras obtidas sob mata e sob pastagem puderam ser nitidamente isoladas dos demais grupos pelo modelo discriminante (Figura 1). Observou-se certa superposição entre os grupos banana, café e cultivo anual, indicando haver semelhança entre os atributos relativos à matéria orgânica nos solos sob esses usos. Esses três últimos grupos têm em comum um histórico de uso (próximo) de fertilizantes minerais e corretivos, e esses insumos não são (ou foram) usados na Mata nem na pastagem, conforme Reis (2002) relatou. Alterações na agregação em função do cultivo e o efeito do uso de fertilizantes e corretivos, aumentando a concentração de bases trocáveis no solo, podem estar sendo responsáveis pela biodegradação das frações menos estáveis da matéria orgânica, representada pelos ácidos fúlvicos, conforme Guggenberger e Zech (1994) comentaram.

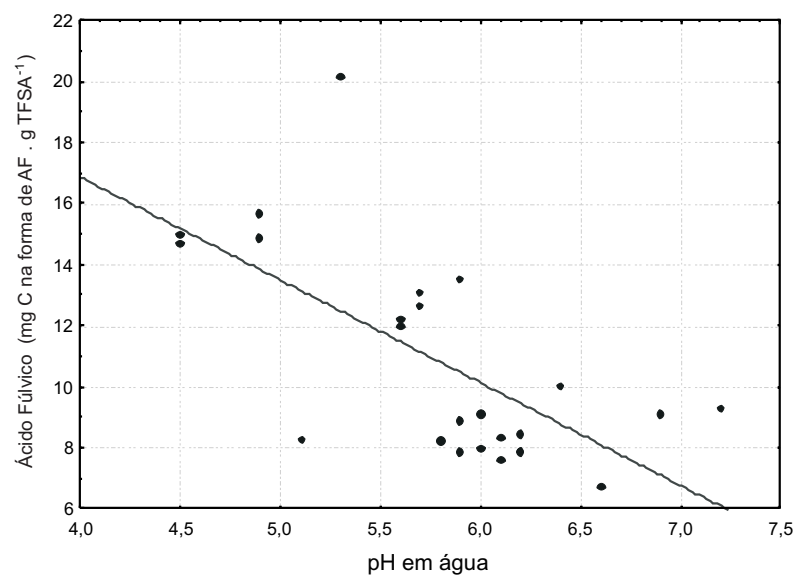

Figura 2 - Gráfico da correlação entre o teor de carbono na forma de ácido fúlvico e o $\mathrm{pH}$ dos solos sob diferentes usos $(\mathrm{n}=25)$.

Figure 2 - Correlation graph between fulvic acid organic carbon and soil $p H$ in soils over different uses $(n=25)$. 


\subsection{Características químicas e físicas dos solos sob diferentes usos}

Os maiores valores médios de carbono orgânico e de nitrogênio total foram encontrados nos solos sob florestas seguidos dos solos sob pastagem (Tabela 2). Embora sejam ambientes bem distintos quanto à dinâmica de deposição e decomposição de resíduos e microclima, a manutenção da estrutura física desses solos parece ser a responsável pela manutenção dos teores de $\mathrm{C}$ (e $\mathrm{N}$ ) elevados, pois permite que haja diminuição do turnover de macroagregados e, consequentemente, maior estabilização e estocagem de C do solo (DENEF et al., 2008).

Fertilidade maior, no entanto, foi encontrada nos solos sob uso agrícola (cultivo anual, café e banana), com os maiores contrastes, entre os diferentes usos, no teor de fósforo do solo (Tabela 1). Como citado anteriormente, áreas sob uso agrícola (anual ou perene) têm recebido contínua aplicação de adubos, além do aporte significativo de elementos como K, Ca e Mg via cinzas resultantes da queima da capoeira para a limpeza do terreno (REIS, 2002; RESENDE e CAMPELLO, 2004). Embora o aumento da fertilidade do solo no Cerrado se reverta em aumento da produção líquida de biomassa local e dos teores e estoques de C do solo (SILVA et al., 2004) nas áreas amostradas, sob uso agrícola, foi observado exatamente o oposto (relação inversa entre fertilidade e teor de frações de ácidos húmicos) (Tabela 4). Sugere-se que esteja havendo efeito sinérgico da ruptura dos agregados e exposição de diferentes compartimentos da MOS a uma “explosão de decompositores” induzidas pela melhoria dos níveis de fertilidade e acidez do solo. Kuiters e Mulder (1993) mostraram que a concentração e o tipo de cátions disponíveis na solução do solo estão intimamente relacionados com a mobilidade dos compostos orgânicos dissolvidos. Os maiores teores de substâncias húmicas, principalmente de ácidos fúlvicos (Tabela 2) nas áreas de Mata e pastagem e a relação inversa entre o teor de carbono na forma de ácidos fúlvicos e o pH do solo (Figura 2 e Tabela 4), corroboram essa hipótese, pois, segundo Kennedy et al. (1996), a solubilidade das substâncias húmicas aumenta com o incremento do $\mathrm{pH}$ do meio, resultando, assim, em maior suscetibilidade à perda por lixiviação ou escoamento superficial.

Os solos sob uso agrícola apresentaram relação $\mathrm{C} / \mathrm{N}$ ligeiramente inferiores aos solos com Mata e sob pastagem, sugerindo maior grau de humificação das frações orgânicas remanescentes no solo, após sua perturbação com o uso. Essa hipótese deve ser analisada com cautela, pois, embora possa haver perda seletiva de carbono em relação ao nitrogênio nesses sistemas mais intensivos de uso, pode também ser reflexo do uso de fertilizantes nitrogenados. Pela metodologia utilizada neste trabalho não foi possível identificar qual dos dois processos teve maior efeito sobre a relação $\mathrm{C} / \mathrm{N}$ nesses solos.

Embora o pousio represente alternativa agroflorestal viável para manutenção da qualidade do solo na região (BALIEIRO et al., 2005), o uso agrícola desses solos tem levado a alterações significativas na qualidade da matéria orgânica do solo. Dos atributos estudados, os ácidos fúlvicos foram àqueles capazes de discriminar com eficiência os usos distintos do solo em áreas do bioma Mata Atlântica.

\section{CONCLUSÕES}

- O teor de ácidos fúlvicos foi o atributo de maior peso relativo nos modelos de discriminação dos sítios amostrados, sugerindo que o fracionamento de substâncias húmicas permite a observação de alterações no solo que não são possíveis de serem identificadas pela simples determinação do teor de carbono orgânico total.

- Áreas sob uso agrícola apresentaram os menores teores de C, N e de ácidos húmicos, embora com maior fertilidade, evidenciando que a não intervenção no solo (via preparo ou correção/adubação) ou pousio seja componente importante de manejo para a conservação da matéria orgânica do solo em sistema de agricultura migratória na região Serrana do Estado do Rio de Janeiro.

\section{AGRADECIMENTOS}

À FAPERJ, pelo auxílio ao desenvolvimento deste trabalho, por meio do projeto "Caracterização de substâncias húmicas em solos sob Mata Atlântica no Estado do Rio de Janeiro”, registrado sob o no E-26/171.563/2002. As análises foram realizadas no Laboratório de Matéria Orgânica do Solo da Embrapa Solos, que contou com o apoio financeiro do projeto PRODETAB 036 - 02/01 do Banco Mundial. As viagens e a coleta de amostras tiveram o apoio do projeto "Geotecnia Ambiental” (PRONEX CNPq/FAPERJ), coordenado pelo Departamento de Engenharia Civil da PUC Rio.

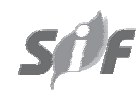

Revista Árvore, Viçosa-MG, v.34, n.4, p.685-690, 2010 


\section{REFERÊNCIAS}

BALIEIRO, F. C. et al. Qualidade do Solo em Áreas Degradadas. In: CONGRESSO BRASILEIRO DE CIÊNCIAS DO SOLO, 30., 2005, Recife. Anais... Recife: UFRPE, Embrapa Solos, SBCS, 2005. CD ROM.

BENITES, V. M., MADARI, B., MACHADO, P. L. O. A. Extração e fracionamento quantitativo de substâncias húmicas do solo: Um procedimento simplificado de baixo custo. Rio de Janeiro: 2003. 13p. (Embrapa Solos. Comunicado Técnico, 14).

BURROUGH, P. A.; WEBSTER, R. Improving a reconnaissance soil classification by multivariate methods. Journal of Soil Science, v.27, n. 4, p.554-571, 1976.

DENEF, K. et al. Microaggregate-associated carbon as a diagnostic fraction for managementinduced changes in soil organic carbon in two Oxisols. Soil Biology \& Biochemistry, v.39, n.5, p.1165-1172, 2007.

EMPRESA BRASILEIRA DE PESQUISA AGROPECUÁRIA - EMBRAPA. Manual de métodos de análise de solo. Rio de Janeiro: Embrapa/SNLCS, 1997. 212p.

GREGORICH, C. M. R. et al. Towards a minimum data set to assess soil organic matter quality in agricultural soils. Canadian Journal of Soil Science, v.74, n.4, p.367-385, 1994.

GUGGENBERGER, G.; ZECH, W. Dissolved organic carbon in florest floor leachates: simple degradation products or humic substances? Science Total Enviroment, v.152, n.1, p.37-47, 1994.

HAYNES, R. J. Labile organic matter as an indicator of organic matter quality in arable and pastoral soils in New Zealand. Soil Biology \& Biochemistry, v.32, n.2., p.211-219, 2000.

KENNEDY, J.; BILLETT, M. F.; DUTHIE, D. Organic matter retention in an upland humic Podzol; the effects o pH and solute type. European Journal of Soil Science, v.47, n.4, p.615-625, 1996.
KUITERS, A. T.; MULDER, W. Water-soluble organic matter in forests soils: complexing properties and implications for soil equilibria. Plant and Soil, v.152, n.2,p.215-224, 1993.

MANLY, B. F. J. Multivariate statistical methods: a primer. Boca Raton: Chapman \& Hall.. 1994, 215p.

NORRIS, J.M. The application of multivariate analisys to soil studies. I Grouping soils using different properties. Journal of Soil Science, v. 22, n.1, p.69-80, 1971.

RESENDE, A. S., CAMPELLO, E. F. C. Seminário sobre agricultura migratória na região serrana do Rio de Janeiro. Seropédica: Embrapa Agrobiologia, 2004. 92p. (Embrapa Agrobiologia. Cadernos de Ecologia, Série Especial).

RESENDE, M.; LANI, J. L.; REZENDE, S. B. Pedossistemas da Mata Atlântica: considerações pertinentes sobre a sustentabilidade. Revista Árvore., v.26, n.3, p.261-269, 2002.

REIS, L. L. Sistema de agricultura migratória na região serrana do Estado do Rio de Janeiro: avaliação de indicadores de sustentabilidade. $\quad 2002.128 f$. Dissertação (Mestrado em Ciência do Solo) - Universidade Federal Rural do Rio de Janeiro, Seropédica, 2002.

STATSOFT. Statistica for Windows: user guide. Statsoft Incorporation, 1999. 293p.

STEVENSON, F. J. Humus chemistry. New York: Wiley, 1996. 443p.

SILVA, J. E. et al. Carbon storage in clayey Oxisol cultivated pasture in the "Cerrado" region, Brazil. Agriculture, Ecosystems and Environment, v.103, p.357-363, 2004.

TÓTOLA, M. R.; CHAER, G. M. Microrganismos e processos microbiológicos como indicadores da qualidade dos solos. Tópicos Avançados em Ciência do Solo, v.2, p.195-276, 2002. 